\title{
Variations saisonnières de l'activité de la phosphatase alcaline des communautés planctoniques bactériennes et algales dans le lac réservoir Allal El Fassi, Maroc
}

\author{
S.M. Raoui ${ }^{1}$ \\ S. Rachiq ${ }^{2}$ \\ N. Chadli ${ }^{2}$ \\ M. Alaoui Mhamdi ${ }^{1}$
}

Mots-clés : bactéries, phytoplancton, activité phosphatase alcaline, variations saisonnières, réservoir.

Les variations verticales et saisonnières de l'activité de la phosphatase alcaline (APA) des communautés microplanctoniques ont été étudiées dans le lac réservoir mésotrophe Allal El Fassi, situé sur l'oued Sebou. Les échantillons ont été collectés à différentes profondeurs au point le plus profond du lac, de mars à décembre 1998. Les résultats montrent que l'APA totale est exclusivement associée à la fraction de taille 0,22-100 $\mu \mathrm{m}$ dont les valeurs moyennes sont comprises entre 0,107 et 1,780 mmol PNP $\mathrm{l}^{-1} \mathrm{~h}^{-1}$. La fraction dissoute $(<0,22 \mu \mathrm{m})$ ne présente aucune activité liée à cette enzyme. Au niveau des couches superficielles, plus de $60 \%$ de l'APA totale sont corrélés à la fraction de taille $0,22-0,65 \mu \mathrm{m}$ à majorité bactérienne. Cependant, avec la profondeur, l'APA est plutôt associée à la fraction de taille $0,65-100 \mu \mathrm{m}$ à dominance phytoplanctonique ( $>58 \%$ de l'APA totale). Cette activité liée aux algues est significativement corrélée avec la densité phytoplanctonique et avec les concentrations de la chlorophylle $a$. L'absence de corrélation significative entre l'APA et les teneurs en phosphore total et orthophosphates suggère une indépendance entre l'APA et les orthophosphates.

Seasonal variations of the alkaline phosphatase activity from the bacterial and algal planktonic communities in the mesotrophic reservoir lake Allal El Fassi, Morocco

Keywords : bacteria, phytoplankton, alkaline phosphatase activity, seasonal variations, reservoir.

Seasonal and vertical variations of alkaline phosphatase activity (APA) of the bacterial and algal planktonic communities have been studied in the mesotrophic Allal El Fassi reservoir located on the Sebou river. Samples were collected at differents depths

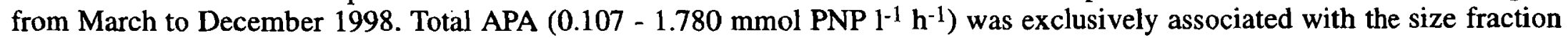
0.22-100 $\mu \mathrm{m}$. Free APA associated with the dissolved fraction $(<0.22 \mu \mathrm{m})$ was not detected. In the superficial layers, more than $60 \%$ of APA was correlated with the $0.22-0.65 \mu \mathrm{m}$ size fraction. In deeper layers, more than $58 \%$ of total APA was associated with 0.65-100 $\mu \mathrm{m}$ size fraction and was significantly correlated with algal parameters (algal counts, chlorophyll $a$ ). The lack of correlation between phosphorus (P-PO ${ }_{4}^{3-}$ and P-PT) and total APA suggests the independance of phosphorus to APA.

\section{Introduction}

La retenue de barrage Allal El Fassi est un réservoir où les processus d'eutrophisation sont menaçants (Bouhaddioui 1997, Jabari 1998). Cette retenue est l'un des réservoirs marocains dont le bassin versant est

1. Faculté des Sciences Dhar Mehrez, Département de biologie, B.P. 1796, Fès, Maroc.

E-Mail : siraoui@yahoo.fr

2. F.S.T., Département des Sciences de la Vie, B.P. 2202, Fès Säss, Fès, Maroc. soumis à une forte activité agricole. Des quantités importantes d'éléments fertilisants pourraient être acheminées vers la retenue d'eau par le lessivage du sol, ce qui se répercuterait sur la qualité de l'eau (Loudiki 1990, Alaoui 1993). Avec l'amplification du phénomène d'eutrophisation des eaux stagnantes naturelles ou artificielles, une attention grandissante a été accordée à l'étude des processus de recyclage du phosphore, longtemps reconnu comme facteur limitant de la prolifération algale en milieux aquatiques (Devaux 1977, Aleya et al. 1994). L'activité phosphatasique est un mécanisme important pour la libération du phosphore 
minéral dissous $\left(\mathrm{P}_{-} \mathrm{PO}_{4}{ }^{3-}\right)$ à partir des composés organo-phosphorés. De plus, la phosphatase alcaline est souvent considérée comme un bon indicateur en condition de déficience en phosphore (Healey \& Hendzel 1979, Gage \& Gorham 1985, Petterson 1980). Cette activité phosphatasique a également été proposée par Jones (1972) comme un indicateur du degré d'eutrophisation des milieux lacustres. Cette enzyme peut être libérée dans le milieu par excrétion, en réponse à un déficit en orthophosphate ou par lyse résultant de la prédation exercée par le zooplancton sur les bactéries et les algues (Jansson et al. 1988). De nombreux travaux portant sur l'activité de la phosphatase alcaline (APA), associée à la communauté planctonique bactérienne et algale ont été réalisés dans des milieux aquatiques (e.g. Jamet et al. 1997, Kalinowska 1997). L'existence d'une forte compétition entre les bactéries et le phytoplancton pour l'utilisation du phosphore minéral (Currie \& Kalff 1984a) a posé plusieurs questions concernant l'origine de l'APA. Stewart \& Wetzel (1982) attribuent la majorité de l'APA aux bactéries, alors que Berman (1970) et Kalinowska (1997) montrent que cette APA est liée en majorité au phytoplancton. Le présent travail a pour objectif l'étude des variations saisonnières de l'activité potentielle de la phosphatase alcaline et la contribution relative des populations planctoniques bactériennes et algales à cette activité.

\section{Matériel et méthodes}

Le réservoir Allal El Fassi est un lac monomictique chaud mésotrophe (Bouhaddioui 1997), situé sur l'oued Sebou à $47 \mathrm{~km}$ de la ville de Fès (Fig. 1). Mis en eau depuis 1992, il a un bassin versant de $5570 \mathrm{~km}^{2}$ et un volume de $8110^{6} \mathrm{~m}^{3}$ d'eau et une profondeur max. de $30 \mathrm{~m}$. Les eaux de cette retenue sont destinées à l'irrigation et à l'alimentation des galeries de Matmata, assurant un transfert des eaux de l'oued Sebou vers le lac Idriss $\mathrm{I}^{\mathrm{er}}$.

Les prélèvements bimensuels d'eau destinés aux analyses des facteurs biotiques et abiotiques ont été effectués du 27 mars au 21 décembre 1998 à l'aide d'une bouteille de type Van Dorn horizontale en PVC opaque, au point le plus profond du lac, à cinq profondeurs : $0 \mathrm{~m},-2 \mathrm{~m},-5 \mathrm{~m},-10 \mathrm{~m}$, et à $-1 \mathrm{~m}$ du fond.

$\mathrm{La}$ température, l'oxygène dissous, $\mathrm{P}-\mathrm{PO}_{4}{ }^{3-}$ et $\mathrm{P}-\mathrm{PT}$ ont été analysés selon les méthodes standards reprises chez Golterman (1978) et Rodier (1984).

Les abondances bactériennes ont été déterminées en microscopie à épifluorescence selon le protocole proposé par Hobbie et al. (1977), après marquage au DAPI (4'-6-diamidino-2-phénylindol dihydrochlorure, Sigma) selon Porter \& Feig (1980). La densité des bactéries fixées a été déterminée par comptage des cellules bactériennes attachées sur la face visible du substrat multiplié par deux pour prendre en considéra-
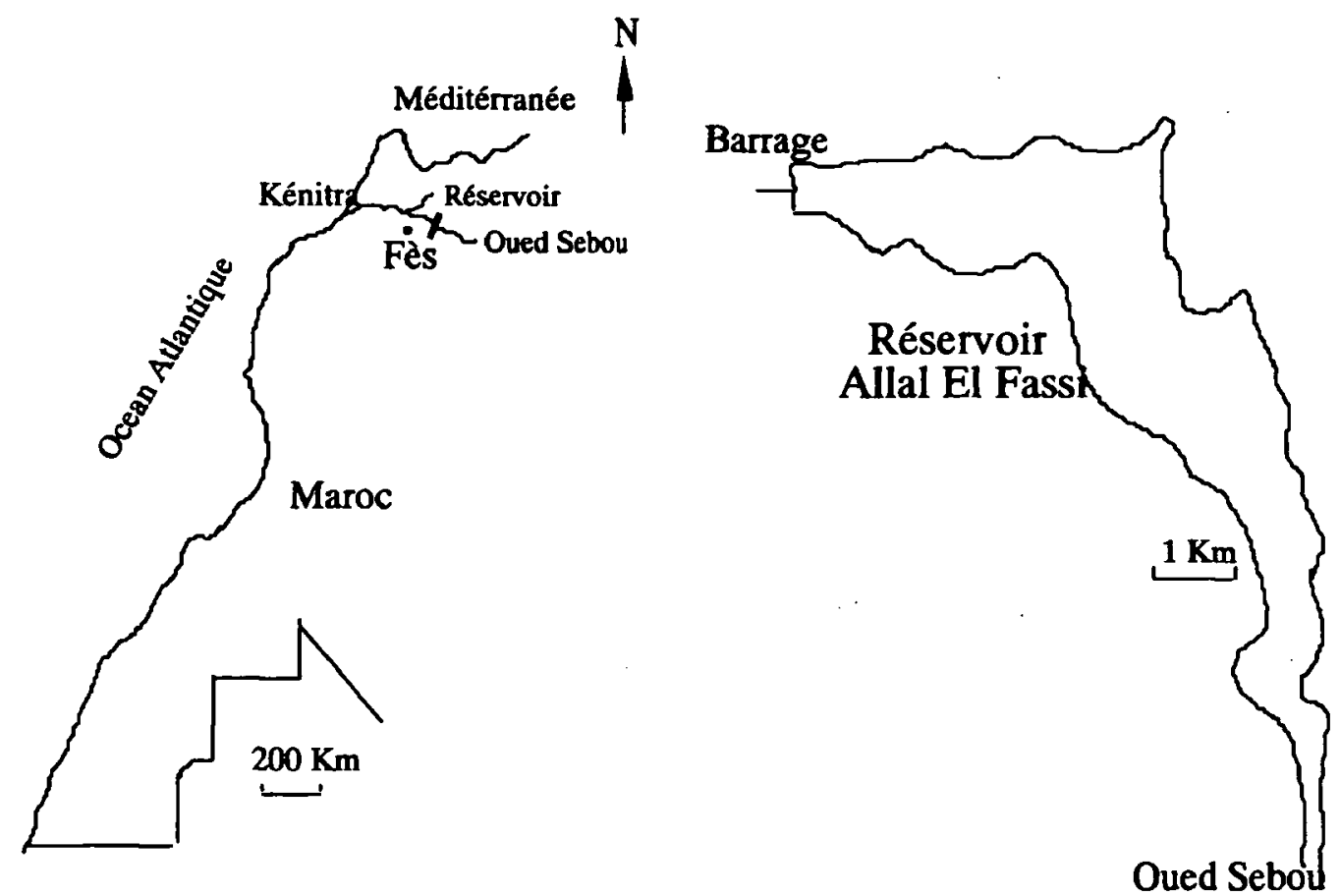

Fig. 1. Localisation géographique du réservoir Allal El Fassi.

Fig. 1. Geographic location of the Allal El Fassi reservoir. 
tion la fixation sur la face cachée du substrat. La biomasse bactérienne a été déterminée par la conversion en carbone des biovolumes bactériens déterminés. Le facteur de conversion utilisé est celui proposé par Bratback (1985) et Lee \& Furhman (1987).

Les cellules phytoplanctoniques ont été dénombrées à l'aide d'un microscope inversé (Utermöhl 1958), méthode modifiée par Legendre \& Watt (1971-1972). La biomasse algale, exprimée en carbone, a été calculée à partir d'un biovolume cellulaire moyen de chaque espèce conformément à la méthode de Lohman (1908). La chlorophylle $a$ a été extraite à l'acétone $90 \%$ et le dosage réalisé selon SCOR-UNESCO (1966), d'après Aleya \& Devaux (1989).
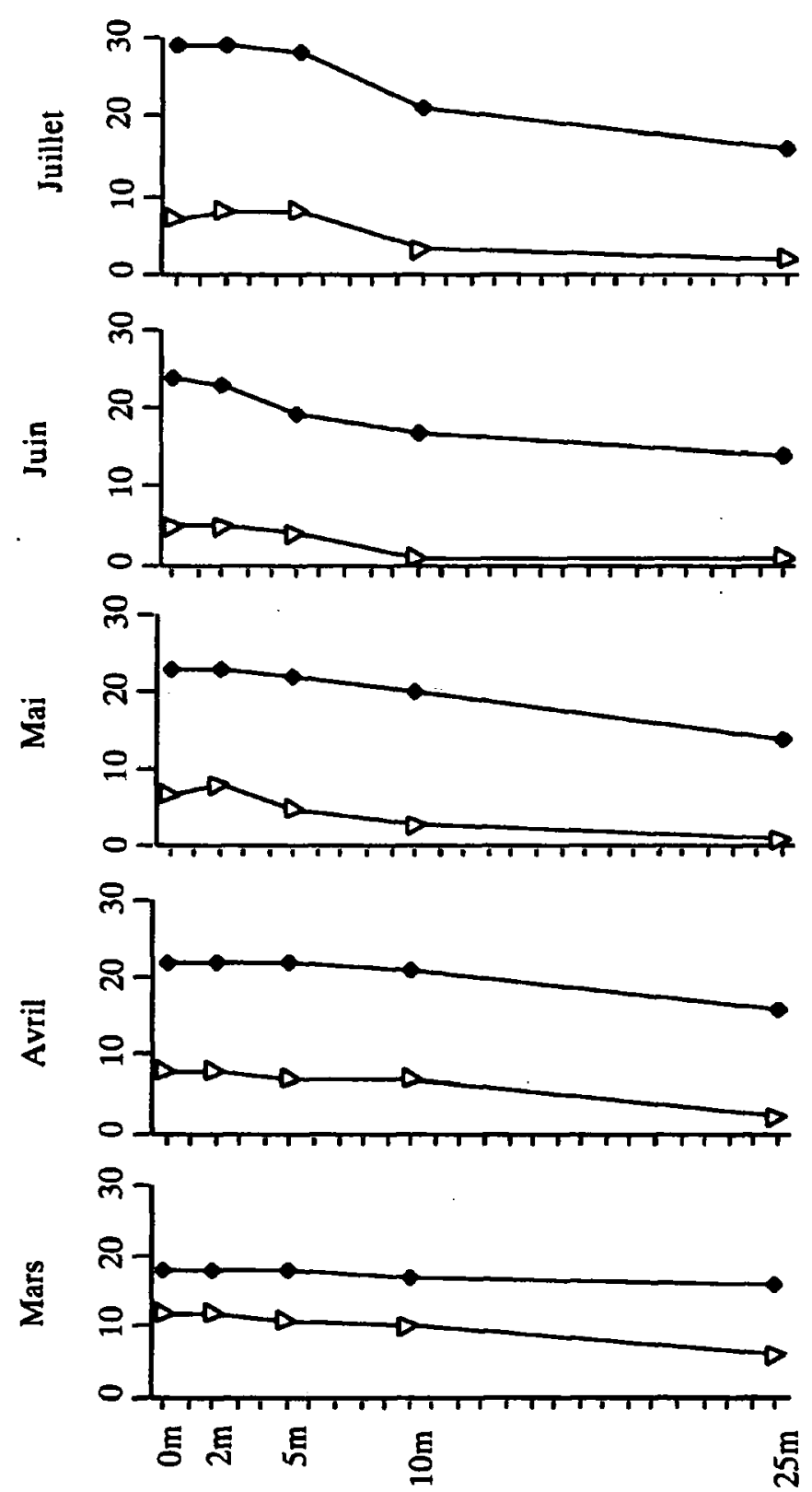

Afin de supprimer la majorité des particules (vivantes ou non) de grande taille, une préfiltration à travers $100 \mu \mathrm{m}$ a été réalisée. Des échantillons de $100 \mathrm{ml}$ prélevés dans des flacons stériles ont immédiatement été filtrés sous vide modéré $(<100 \mathrm{~mm} \mathrm{Hg})$ à travers des membranes Nuclépores de porosité $0,22 \mu \mathrm{m}$ et $0,65 \mu \mathrm{m}$. Les filtres placés dans des tubes à hémolyse stériles ont été immergés dans $3 \mathrm{ml}$ de tampon (Tris$\left.\mathrm{HCl} 0,1 \mathrm{M} ; \mathrm{Mg}^{++} 10^{-3} \mathrm{M} ; \mathrm{pH} 8,5\right)$ contenant $1 \mathrm{mg}$. $\mathrm{ml}^{-1}$ de p-Nitrophényl phosphate (p-NPP). Le dosage de l'APA libre a été réalisé sur un volume de filtrat de $100 \mathrm{ml}$ à travers $0,22 \mu \mathrm{m}$. Après incubation à $37^{\circ} \mathrm{C}$ sous une légère agitation pendant 6 heures, la lecture spectrophotométrique a été réalisée à $410 \mathrm{~nm}$ (Rei-
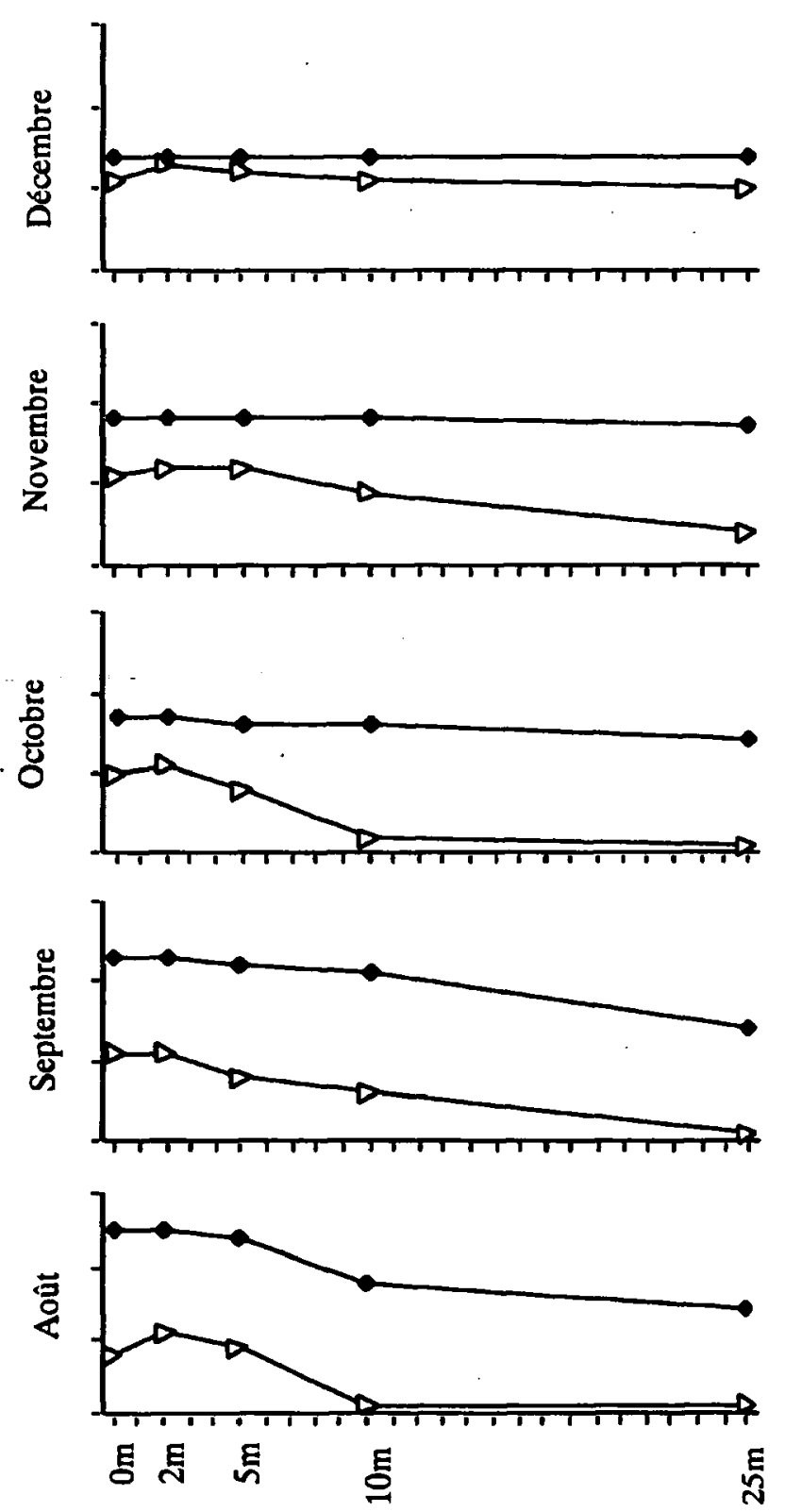

Profondeurs (m)

Fig. 2. Variations spatio-temporelles de la température de l'eau $\left({ }^{\circ} \mathrm{C}\right)(-\bullet-)$ et des concentrations en oxygène dissous (mg. $\left.\mathrm{l}^{-1}\right)(-\nabla-)$ au niveau de la colonne d'eau.

Fig. 2. Spatial and temporal variations of temperature $\left({ }^{\circ} \mathrm{C}\right)(-\bullet)$ and dissolved oxygen concentrations (mg. $\left.\mathrm{I}^{-1}\right)(-\nabla \longrightarrow)$ at different depths. 
chardt et al. 1967). L'incubation a été réalisée à $37^{\circ} \mathrm{C}$ pour obtenir une activité potentielle maximale conformément à ce qui est pratiqué dans les milieux aquatiques stagnants (Kalinowska 1997, Jamet et al. 1997).

Les résultats de l'activité potentielle de la phosphatase alcaline (APA) sont exprimés en mmol p-Nitrophénol (mmol PNP) libérés par litre et par heure pour trois fractions de taille, 0,22-100 $\mu \mathrm{m}$ (APA totale), 0,65-100 $\mu \mathrm{m}$ (APA liée au phytoplancton) et une fraction de 0,22-0,65 $\mu \mathrm{m}$ (APA liée aux bactéries). L'activité pour cette dernière fraction est obtenue par soustraction entre l'APA totale et celle associée au phytoplancton.

\section{Résultats}

\section{1. Analyses physique et chimique}

La température de $1^{\prime}$ eau a varié de $12^{\circ} \mathrm{C}$ près du fond durant le mois de juin à $29^{\circ} \mathrm{C}$ à la surface en finjuillet (Fig. 2). La stratification thermique des eaux s'est étalée du printemps à la fin de l'été. Les concentrations en oxygène dissous, au niveau de l'épilimnion, ont fluctué entre 4 et $12 \mathrm{mg} . \mathrm{1}^{-1}$. Ces teneurs chutaient en profondeur et devenaient quasiment nulles près du fond durant la période estivale (Fig. 2). Les concentrations en orthophosphates s'annulaient à plusieurs reprises au niveau supérieur de la colonne d'eau et présentaient un maximum de $0,15 \mathrm{mg}^{-1} \mathrm{l}^{-1}$ enregistré au niveau du fond (Fig. 3). Les concentrations en phosphore total (P-PT) étaient comprises entre 0,001 et 0,49 mg P-PT. $1^{-1}$, ce maximum était enregistré en septembre près du fond (Fig. 3 ).

\section{2. Abondance et biomasse bactériennes}

Les abondances bactériennes ont varié de $1,0110^{6}$ bactéries $\mathrm{ml}^{-1}$ le 24 août près du fond à $9,8610^{6}$ bactéries. $\mathrm{ml}^{-1}$ le 17 avril à - $10 \mathrm{~m}$ (Fig. 4). Généralement nous n'avons pas observé de grandes variations entre le niveau $0 \mathrm{~m}$ et - $10 \mathrm{~m}$ (Tableau 1). Quelle que soit la profondeur considérée, les variations saisonnières de la densité bactérienne ont fait apparaître une diminution pendant la période estivale suivie d'une augmentation plus au moins importante selon la profondeur. Les bactéries fixées représentaient en moyenne $16 \%$ de la densité bactérienne totale. Ce pourcentage serait en relation avec la sédimentation des cellules algales sénescentes et les débris. Calculées à partir des biovolumes, les valeurs de biomasses bactériennes ont varié entre $0,22 \mu \mathrm{g} \mathrm{C} .1^{-1}$ à la surface de l'eau et $0,91 \mu \mathrm{g} \mathrm{C}$. $1^{-1}$ à - $10 \mathrm{~m}$ (Tableau 1). La valeur moyenne annuelle a été de $0,57 \mu \mathrm{g} \mathrm{C.} \mathrm{1}^{-1}$.

\subsection{Densité et biomasse phytoplanctoniques}

Les densités phytoplanctoniques ont varié de 1,69 $10^{5}$ cellules. $1^{-1}$ au fond et $9,6010^{5}$ cellules. $1^{-1}$ à $-2 \mathrm{~m}$ (Tableau 1). Les valeurs les plus élevées de densité phytoplanctonique sont généralement observées à la surface et on note une diminution progressive des densités en fonction de la profondeur. Calculées à partir des biovolumes spécifiques moyens, les biomasses
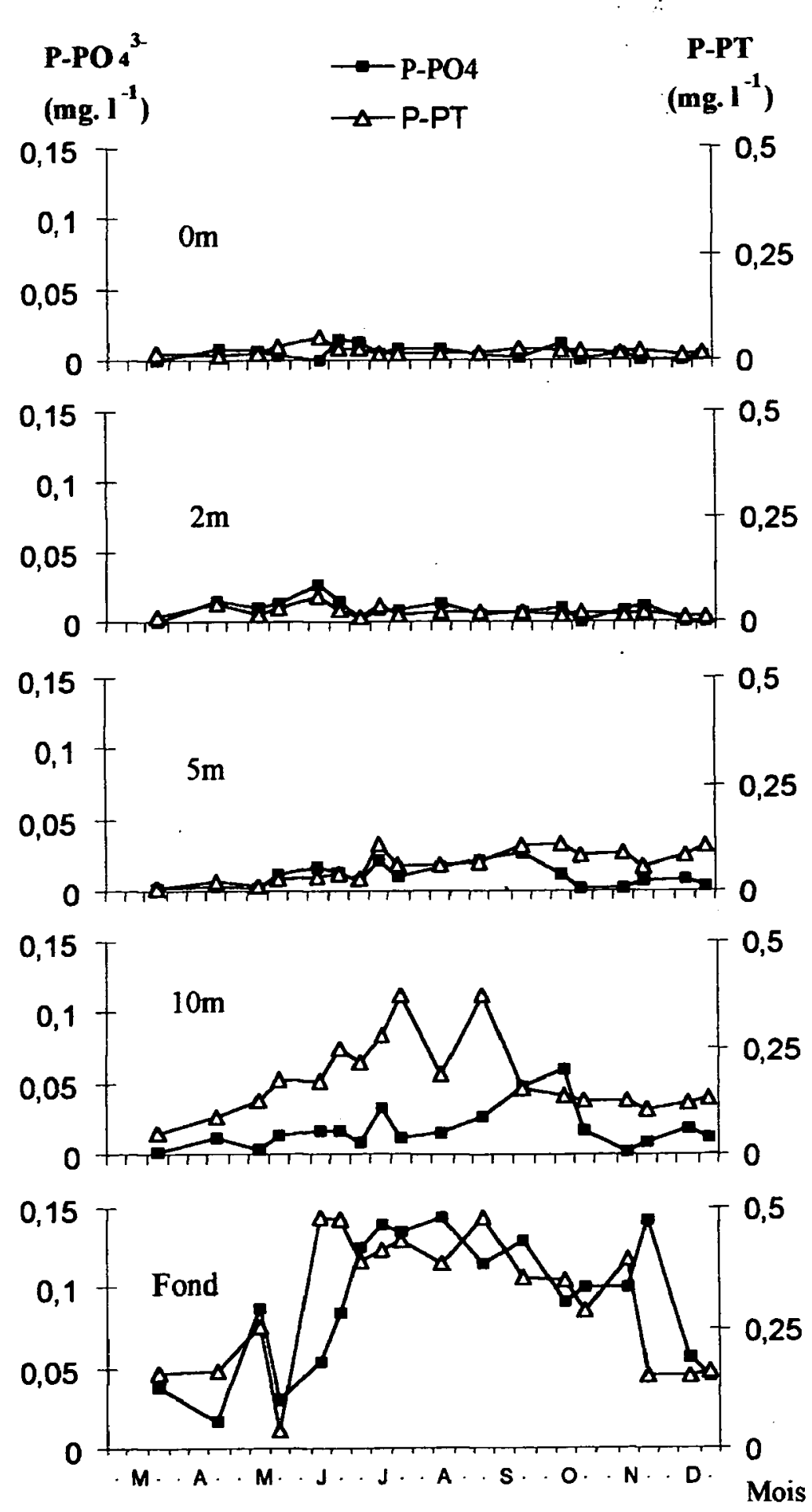

Fig. 3. Variations spatio-temporelles des concentrations en phosphore total et des orthophosphates au niveau de la colonne d'eau.

Fig. 3. Spatial and temporal variations of total phosphorus and orthophosphate concentrations at different depths. 
phytoplanctoniques ont suivi globalement la même évolution que les valeurs de densité et ont montré une grande amplitude de variation de $8,40 \mu \mathrm{g} \mathrm{C} . \mathrm{I}^{-1}$ au fond et $69,30 \mu \mathrm{g} \mathrm{C}$. $\mathrm{l}^{-1}$ à $-2 \mathrm{~m}$ (Tableau 1). Les plus fortes biomasses ont été enregistrées au printemps et en été. Ceci serait lié à la dominance de la Diatomophycée Cyclotella ocellata. Cette espèce était accompagnée de populations, d'importance moindre, de Cryptomonas ovata, Peridinium cinctum. En période automnale, les

\section{Abondances bactériennes}

(x $10^{6}$ bactéries. ml ${ }^{-1}$ )

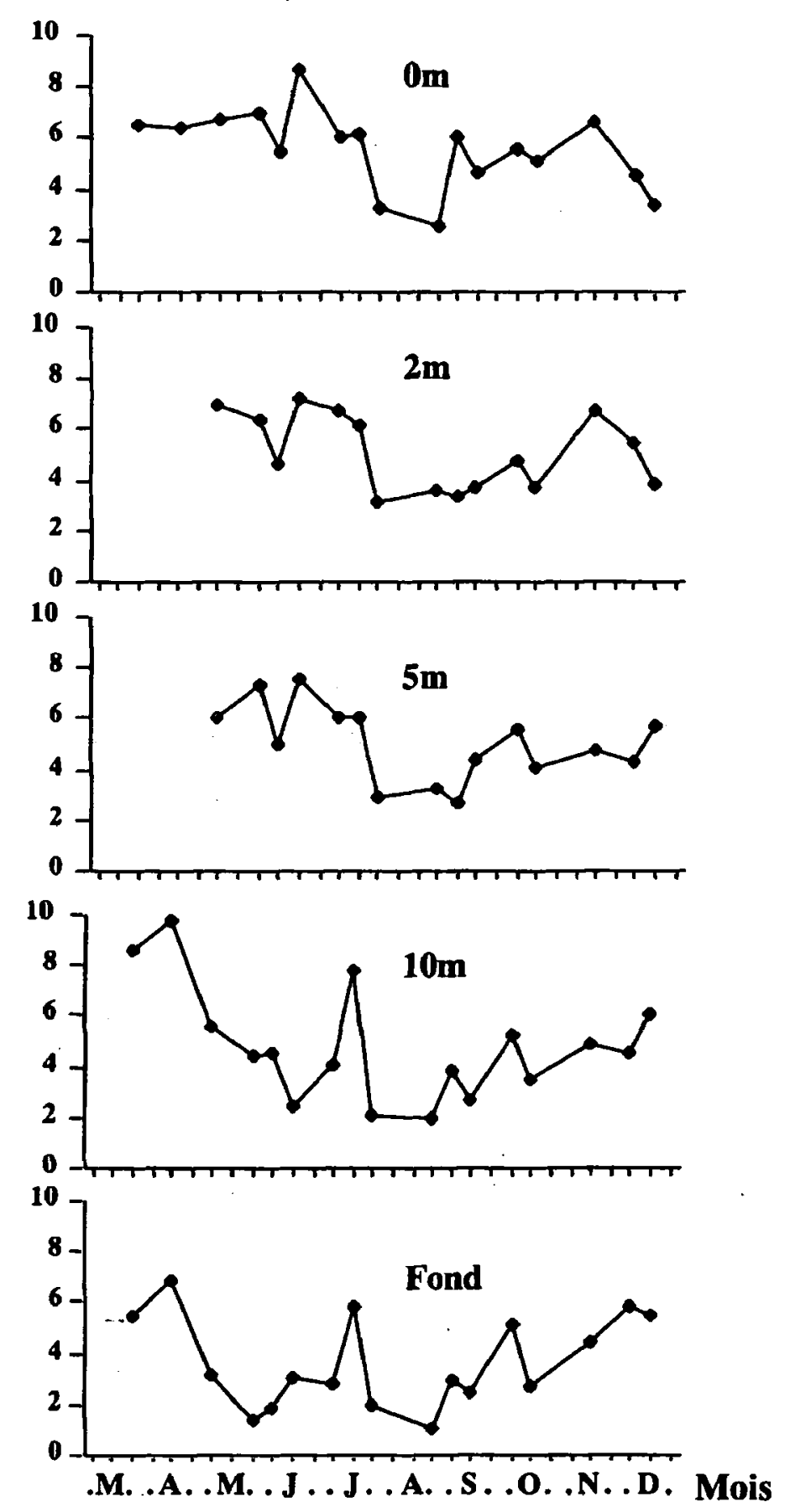

Fig. 4. Variations spatio-temporelles des abondances bactériennes à différentes profondeurs.

Fig. 4. Spatial and temporal variations of the bacterial abundance at different depths. fortes biomasses coïncident avec le développement important de Dinobryon sertularia, Cryptomonas ova$t a$ et Peridinium cinctum. Les concentrations en chlorophylle $a$ s'annulent à plusieurs reprises au niveau du fond et montrent un maximum de $10,17 \mu \mathrm{g} . \mathrm{l}^{-1}$ à une profondeur de - $10 \mathrm{~m}$ (Fig. 5).

\subsection{Activité potentielle de la phosphatase alcaline (APA)}

Au cours de cette étude, l'APA liée à la classe de taille $0,22-100 \mu \mathrm{m}$ a varié entre $0,107 \mathrm{mmol}$ PNP. $\mathrm{l}^{-1}$. $\mathrm{h}^{-1}$ enregistrée fin-août au fond et $1,780 \mathrm{mmol}^{\mathrm{PNP}} \mathrm{1}^{-}$ ${ }^{1}$. $\mathrm{h}^{-1}$ à $0 \mathrm{~m}$ fin-juillet (Fig. 6). L'examen du profil vertical de l'APA montre des valeurs plus élevées en surface et qui diminuent avec la profondeur (Tableau 1). Dans notre réservoir, l'APA enregistrée semblait être entièrement associée à la communauté planctonique bactérienne et algale dans la mesure où aucune trace d'activité liée à cet enzyme n'a été détectée dans la fraction dissoute $(<0,22 \mu \mathrm{m})$.

Nous avons noté une corrélation significative $(0,6<$ $r<0,8 ; p<0,05 ; n=18$ ) entre l'APA et la température de l'eau au niveau $0 \mathrm{~m},-2 \mathrm{~m}$ et $-5 \mathrm{~m}$, alors qu'au niveau des couches profondes ces deux paramètres ne sont pas corrélés. L'absence de corrélation significative $(\mathrm{p}>0,05)$ a également été observée entre l'APA et les concentrations en orthophosphates et en phosphore total pour les trois fractions de taille et à toutes les profondeurs échantillonnées.

L'APA associée à la fraction de taille $0,22-0,65 \mu \mathrm{m}$ (essentiellement bactérienne) représentait des pourcentages de l'APA totale qui variaient entre $28 \%$ à $-5 \mathrm{~m}$ le 01 juillet et $88 \%$ le 15 juillet à $-2 \mathrm{~m}$. La classe de taille $0,65-100 \mu \mathrm{m}$ (essentiellement phytoplanctonique) représentait des pourcentages qui ont fluctué entre $18 \%$ enregistré à $-2 \mathrm{~m}$ le 15 juillet et $93 \%$ de l'APA totale près du fond le 09 septembre. La part relative moyenne au niveau $0 \mathrm{~m}$ et $-2 \mathrm{~m}$ est en grande partie bactérienne et représente respectivement $61 \%$ et $60 \%$ de l'APA totale (Tableau 2). A ces profondeurs, nous avons enregistré une corrélation significative $(r=$ 0,57 à $0 \mathrm{~m}$ et $\mathrm{r}=0,60$ à $-2 \mathrm{~m}, \mathrm{p}<0,05, \mathrm{n}=18$ ) entre les abondances bactériennes et l'APA $(0,22-0,65 \mu \mathrm{m})$. Inversement en dessous de - 2 m, l'APA est plutôt à dominance phytoplanctonique, le pourcentage moyen a été de $58 \%$ de l'APA totale (Tableau 2). Nous notons une corrélation significative entre l'APA de cette fraction et la densité phytoplanctonique $(r=0,67$ à $-5 \mathrm{~m}$ et $\mathrm{r}=0,71 \mathrm{a}-10 \mathrm{~m}, \mathrm{p}<0,05, \mathrm{n}=18$ ), et entre cette activité et les concentrations en chlorophylle $a(\mathrm{r}=0,53$ à $-5 \mathrm{~m}$ et $\mathrm{r}=0,55$ à $-10 \mathrm{~m}, \mathrm{p}<0,05, \mathrm{n}=14)$. Que ce soit la fraction de taille $0,22-0,65 \mu \mathrm{m}$ ou $0,65-100 \mu \mathrm{m}$, les valeurs de l'APA sont relativement plus élevées en 
Tableau 1. Valeurs moyennes annuelles des abondances et des biomasses bactériennes, abondances et biomasses algales, concentrations en chlorophylle $a$ et de l'APA totale à différentes profondeurs.

Table 1. Annual mean values of bacterial abundance and biomass, algal abundance and biomass, chlorophyll $a$ concentrations and the total APA at different depths.

\begin{tabular}{|c|c|c|c|c|c|c|}
\hline & $\begin{array}{l}\text { Abond. bact. } \\
\left(\times 10^{6} \text { bact. } \mathrm{ml}^{-1}\right)\end{array}$ & $\begin{array}{l}\text { Biom. bact. } \\
\left(\mu \mathrm{g} . \mathrm{C}^{1-1} \mathrm{1}^{-1}\right)\end{array}$ & $\begin{array}{l}\text { Abond. algale } \\
\left(\times 10^{5} \text { cell. } 1^{-1}\right)\end{array}$ & $\begin{array}{c}\text { Biom. algale } \\
\left(\mu \mathrm{g} . \mathrm{C}^{-1} \mathrm{1}^{-1}\right)\end{array}$ & $\begin{array}{c}\text { Chl } a \\
\left(\mu \mathrm{g} .1^{-1}\right)\end{array}$ & $\begin{array}{c}\text { APA total } \\
\left(\mathrm{mmol} \mathrm{PNP.}^{-1} \cdot \mathrm{h}^{-1}\right)\end{array}$ \\
\hline $0 \mathrm{~m}$ & 5,7 & 0,22 & 7,18 & 57,70 & 3,79 & 0,706 \\
\hline$-2 m$ & 5,10 & 0,60 & 9,60 & 69,30 & 4,79 & 0,696 \\
\hline$-5 m$ & 5,03 & 0,48 & 7,62 & 53,30 & 4,16 & 0,646 \\
\hline$-10 \mathrm{~m}$ & 4,81 & 0,91 & 4,58 & 37,10 & 3,83 & 0,613 \\
\hline Fond $-1 \mathrm{~m}$ & 3,64 & 0,64 & 1,69 & 8,40 & 1,17 & 0,174 \\
\hline
\end{tabular}

Tableau 2. Valeurs de l'APA (min, max) et pourcentages par rapport à l'APA totale des fractions de taille $0,22-0,65 \mu \mathrm{m}$ et $0,65-100 \mu \mathrm{m}$ le long de la colonne d'eau.

Table 2. Maximal and minimal values and percentages of total APA from two size fractions $0.65-0.22 \mu \mathrm{m}$ and $0.65-100 \mu \mathrm{m}$ at different depths.

\begin{tabular}{lllllll}
\hline & \multicolumn{3}{c}{ APA de la fraction } & \multicolumn{3}{c}{ APA de la fraction } \\
& $\min$ & $\max -0,65 \mu \mathrm{m}$ & \multicolumn{3}{c}{$0,65-0,100 \mu \mathrm{m}$} \\
& $\max$ & $\%$ & $\min$ & $\max$ & $\%$ \\
\hline $0 \mathrm{~m}$ & 0,036 & 0,329 & 61,3 & 0,022 & 0,205 & 38,7 \\
$-2 \mathrm{~m}$ & 0,050 & 0,221 & 60 & 0,029 & 0,116 & 40 \\
$-5 \mathrm{~m}$ & 0,038 & 0,145 & 42,2 & 0,041 & 0,187 & 57,8 \\
$-10 \mathrm{~m}$ & 0,040 & 0,148 & 41,5 & 0,045 & 0,177 & 58,5 \\
Fond -1 m & 0,002 & 0,025 & 23 & 0,022 & 0,075 & 77 \\
\hline
\end{tabular}

période printanière (moyenne $=0,525 \mathrm{mmol} \mathrm{PNP.1} \cdot{ }^{-1} \cdot \mathrm{h}^{-1}$ ) et estivale (moyenne $=0,798 \mathrm{mmol}$ PNP. $1^{-1} \cdot \mathrm{h}^{-1}$ ) qu'en période automnale (moyenne $=0,409 \mathrm{mmol}$ PNP. $1^{-1} \cdot \mathrm{h}^{-1}$ ) (Fig. 7).

Près du fond du lac, l'APA restait faible pour les deux fractions de taille. Les valeurs enregistrées ont varié de 0,007 à $0,083 \mathrm{mmol}$ PNP..$^{-1} \cdot \mathrm{h}^{-1}$ pour la fraction de taille $0,22-0,65 \mu \mathrm{m}$ et de 0,073 à $0,250 \mathrm{mmol}$ PNP.1-1.h $\mathrm{h}^{-1}$ pour la fraction de taille $0,65-100 \mu \mathrm{m}$.

\section{Discussion}

L'APA totale enregistrée pour le réservoir Allal El Fassi $\left(0,107\right.$ à $\left.1,780 \mathrm{mmol}^{\mathrm{PNP}} \mathrm{l}^{-1} \mathrm{~h}^{-1}\right)$ excède largement celles rapportées dans différents lacs européens (Boavida \& Marque 1995, Kalinowska 1997, Jamet et al. 1997). Les valeurs les plus élevées ont été enregistrées au niveau des couches superficielles, en période estivale, lors de la stratification thermique. Cette augmentation coïncide avec des valeurs élevées de la température enregistrée à ces niveaux ; en effet, l'APA et la température sont significativement corrélées. A l'inverse, au niveau du fond, les faibles valeurs de l'APA seraient liées d'une part, à la baisse de la température et d'autre part, aux faibles densités bactériennes et des cellules phytoplanctoniques sénescentes. Des résultats semblables ont été observés par Wetzel (1981) et Coveney \& Wetzel (1995) dans le lac Lawrence (Michigan, USA).

Si l'APA est souvent considérée comme indicateur de déficience en phosphore (Petterson 1980, Gage \& Gorham 1985), il n'en est pas de même dans cette étude. En effet, aucune corrélation significative n'a été enregistrée entre l'APA et les concentrations en phosphore total ou en orthophosphates suggérant une indé- 

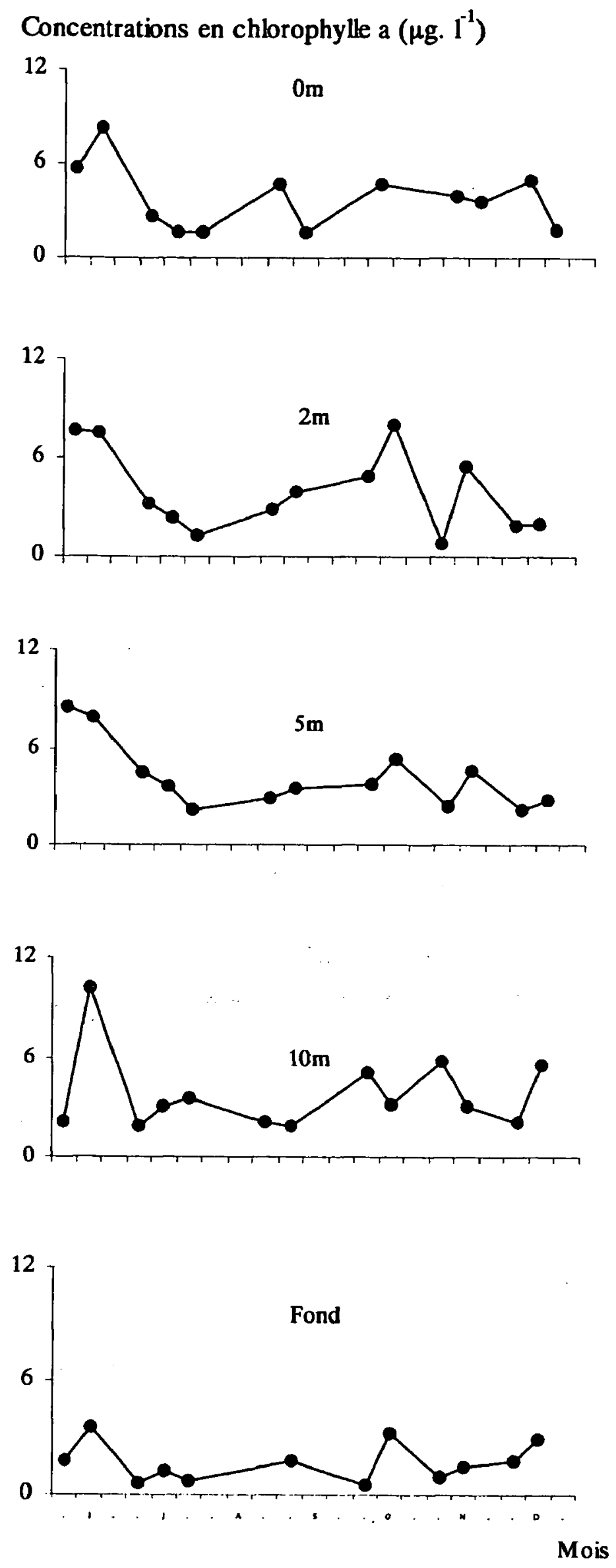

Fig. 5. Variations spatio-temporelles des concentrations en chlorophylle $a$ à différentes profondeurs.

Fig. 5. Spatial and temporal variations of chlorophyll $a$ concentrations at different depths.
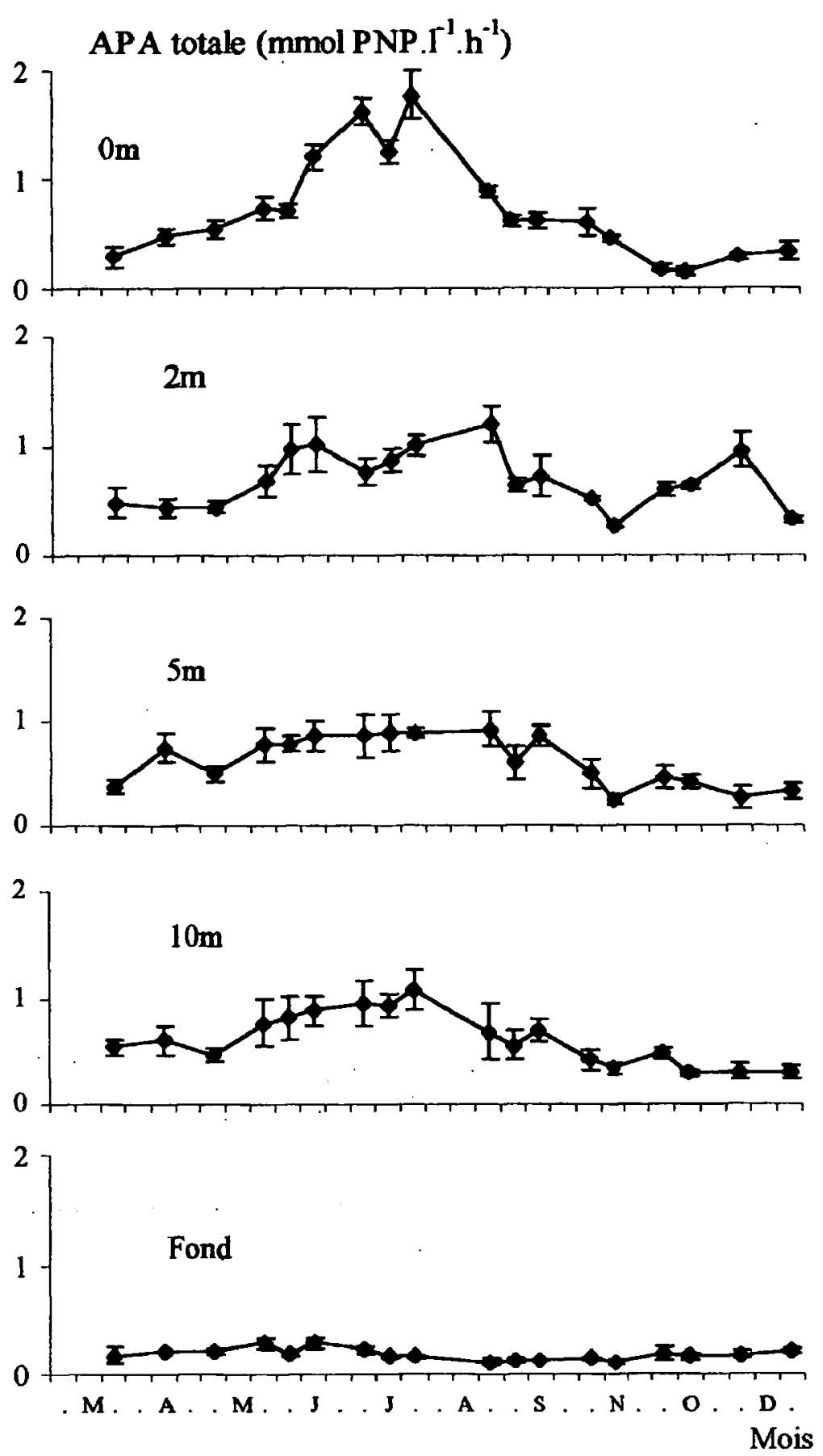

Fig. 6. Variations spatio-temporelles de l'activité de la phosphatase alcaline totale à différentes profondeurs.

Fig. 6. Spatial and temporal variations of total alkaline phosphatase activity at different depths.

pendance probable entre l'APA et la concentration en orthophosphates. L'absence de corrélation entre ces deux paramètres a également été constatée par plusieurs auteurs dans différents milieux (Boavida \& Marque 1995, Jamet et al. 1995, 1997). Cependant, selon Gage \& Gorham (1985) et Hernandez et al. (1993), l'APA serait plutôt corrélée au phosphore intracellulaire des bactéries. Jansson et al. (1988) ont avancé que de fortes APA dans le milieu lacustre sont observées lorsque les concentrations en phosphore sont extrêmement faibles. Au sein du réservoir étudié, les fortes concentrations en orthophosphate enregistrées parallè- 

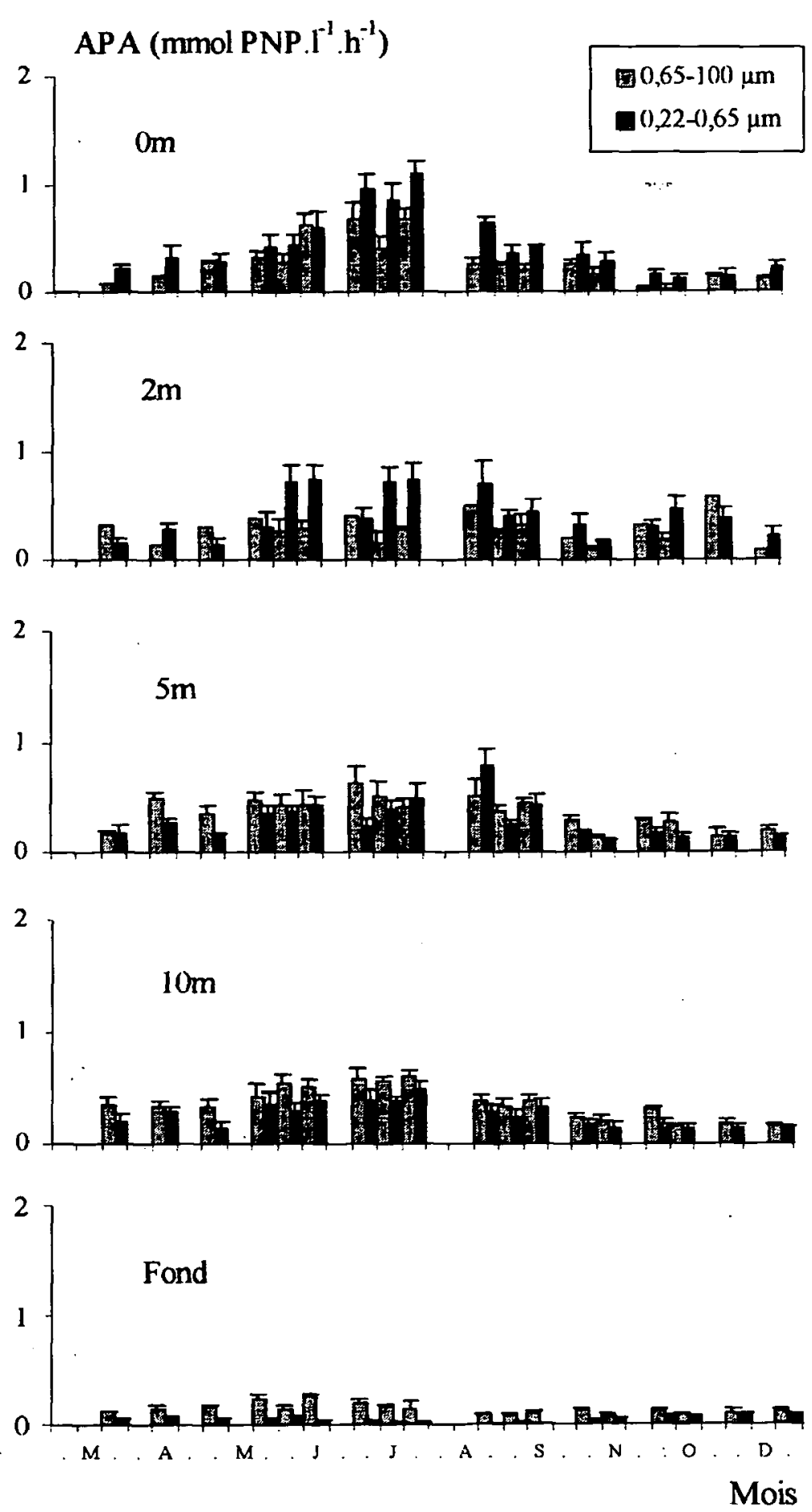

Fig. 7. APA des deux fractions de taille du microplancton à différentes profondeurs.

Fig. 7. APA of two size fractions of microplankton at different depths.

lement à une APA importante suggèrent que la phosphatase alcaline mesurée serait probablement constitutive. On ne peut exclure l'effet inhibiteur du phosphore réactif soluble, ainsi les fortes concentrations en orthophosphate enregistrées près du fond inhiberaient l'APA.

Au niveau de l'épilimnion, l'APA est maximale alors que les concentrations en orthophosphate sont à l'état de trace, résultat probablement d'une assimilation par le bactérioplancton et le phytoplancton (Capblancq 1990).
Conformément aux observations de Francko (1983) et Jamet et al. (1997), l'APA enregistrée dans le réservoir étudié est entièrement liée à la communauté planctonique bactérienne et algale. L'APA liée à la fraction dissoute $(<0,22 \mu \mathrm{m})$ n'a pas été détectée. Ce résultat est en contradiction avec ceux obtenus par Currie et al. (1986) et Kalinowska (1997) qui ont montré que l'APA liée à la fraction dissoute est importante. La non détection de l'APA libre peut s'expliquer par la faible sensibilité de la méthode au p-NPP. Cependant, avec des concentrations en p-NPP identiques à celles que nous avons utilisées, Jamet (1995) a pu détecter cette activité libre dans le réservoir hypereutrophe de Villerest en France.

En fonction de la profondeur, l'APA totale a montré une dominance tantôt bactérienne et tantôt phytoplanctonique. En effet, l'APA de la fraction à majorité bactérienne $(0,22-0,65 \mu \mathrm{m})$ est relativement élevée $(61 \%$ de l'APA totale) en surface. Toutefois, l'importance de l'APA liée aux bactéries diminue avec la profondeur au profit de celle associée à la classe de taille 0,65-100 $\mu \mathrm{m}$ à dominance phytoplanctonique. De plus, les valeurs de l'APA ont augmenté en périodes printanière et estivale. A ces saisons, le phytoplancton est en grande partie dominé par des Diatomophycées centriques représentées par Cyclotella ocellata. Hino (1988) et Olsson (1990) ont montré que les Diatomées et Cyanobactéries contribuaient significativement à l'APA.

Pour des raisons méthodologiques, il est difficile de faire la part relative de l'APA liée aux bactéries fixées et celle des microalgues. L'interférence bactérienne serait négligeable puisque, en terme de carbone, les bactéries fixées ne représentaient que $16 \%$ des bactéries totales soit une fraction infime de la biomasse phytoplanctonique (moyenne $=0,05 \%$ ).

\section{Conclusion}

Les communautés planctoniques bactériennes et algales du réservoir Allal El Fassi contribuent significativement à la totalité de l'APA dont le maximum d'activité est observé à la surface de la colonne d'eau lors de la stratification thermique estivale. En surface $(0$ à $-2 \mathrm{~m}$ ), la fraction de taille $0,22-0,65 \mu \mathrm{m}$ à dominance bactérienne contribue significativement à l'APA totale (>60\%). Au-dessous, cette activité redevient importante pour la fraction de taille $0,65-100 \mu \mathrm{m}$ à dominance phytoplanctonique. L'absence de corrélation significative entre l'APA et les concentrations en orthophosphates suggère une indépendance entre l'APA et les orthophosphates. 


\section{Remerciements}

Nous exprimons nos vifs remerciements au service régional d'hydraulique de la ville de Fès et à tout le personnel de la direction du réservoir Allal El Fassi.

\section{Références}

Alaoui Mhamdi M. 1993. - Intérêt de l'étude des bilans biogéochimiques de l'azote et du phosphore pour la compréhension du déterminisme de l'eutrophisation de quelques retenues marocaines. Thèse de Doct. d'état, Univ. SMBA, Fès : 200 p.

Aleya L. \& Devaux J. 1989. - Interêt et signification écophysiologique de l'estimation de la biomasse et de l'activité photosynthétique de diverses fractions de taille phytoplanctonique en milieu lacustre eutrophe. Rev. Sci. Eau, 2 : 353-37.

Aleya L., Desmolles F., Bonnet M.P. \& Devaux J. 1994. - The deterministic factors of the Microcystis aerogenosa blooms over a biyearly survey in hypereutrophic reservoir of Villerest (Roanne, France). Arch. Hydrobiol., $99: 1-26$.

Berman T. 1970. - Alkaline phosphatases and phosphorus availability in Lake Kinneret. Limnol. Oceanogr., $15: 663-674$.

Boavida M.J. \& Marque R.T. 1995. - Low activity of alkaline phosphatase in two eutrophic reservoirs. Hydrobiologia, 297 : 11-16.

Bouhaddioui A. 1997. - Bilan biogéochimique de l'azote et du phosphore et dynamique des populations phytoplanctoniques de la retenue de barrage Allal El Fassi. Thèse de $3^{\text {eme }}$ cycle. Univ. SMBA. Fac. Sci. Fès : $188 \mathrm{p}$.

Bratback G. 1985. - Bacterial biovolume and biomass estimations. Appl. Environ. Microbiol., 49 : 1488-1493.

Capblancq J. 1990. - Nutrient dynamics and pelagic food web interaction in oligotrophic and eutrophic environments : an overview. Hydrobiologia, $207: 1-14$.

Coveney M.F. \& Wetzel R.G. 1995. - Biomass, production, and specific growth rate of bacterioplankton and coupling to phytoplankton in an oligotrophic lake. Limnol. Oceanogr., 40 (7) : $1187-1200$.

Currie D.J. \& Kalff J. 1984a. - A comparison of the abilities of freshwater algae and bacteria to acquire and retain phosphorus. Limnol. Oceanogr., $29: 298-310$.

Currie D.J., Bentzen E. \& Kalff J. 1986. - Does algal-bacterial phosphorus partitionning vary among lake ? A comparative study of orthophosphate uptake and alkaline phosphatase activity in freshwater. Can. J. Fish. Aquat. Sci., 43: 311-317.

Devaux J. 1977. - Dynamique des populations phytoplanctoniques dans deux lacs du Massif Central français. Thèse de Doct. ès-Sci: naturelles, Clermont Ferrand II : 184 p.

Francko D.A. 1983. - Size fractionation of alkaline phosphatase activity in Lake water by membrane filtration. J. Freshwat. Ecol., $2: 305-309$.

Gage M.A. \& Gorham E. 1985. - Alkaline phosphatase activity and cellular phosphorus as an index of the phosphorus status of phytoplankton in Minnesota Lake. Freshwat. Biol., $15: 227-233$.

Golterman H.L., Clymo R.S. \& Ohstad M.A.M. 1978. - Methods for physical and chemical analyses of freshwater. IBP. Manuel $\mathrm{n}^{\circ} 8$ (2nd edit.). Blackwell Scientific Publication, Oxford : $213 \mathrm{p}$.

Healey F.P. \& Hendzel L.L. 1979. - Indicators of phosphorus and nitrogen deficiency in five algae in culture. J. Fish. Res. Bd. Can., $36: 1369-1369$.

Hernandez I., Fernandez J.A. \& Neill F.X. 1993. - Influence of phosphorus status on the seasonal variation of alkaline phosphatase activity in Porphyra umbilicalis (L.) Kützing. J. Exp. Mar. Biol. Ecol., 173 : 181-196.
Hino S. 1988. - Fluctuation of algal alkaline phosphatase activity and the possible mechanisms of hydrolysis of dissolved organic phosphorus in Lake Barato. Hydobiologia, 157 : 77-84.

Hobbie J.E., Daley R.J. \& Jasper S., 1977. - Use of nucleopore filters for counting bacteria by fluorescence microscopy. Appl. Environ. Microbiol., 33 : 1225-1228.

Jabari A. 1998. - Structure et dynamique des populations zooplanctoniques de la retenue de barrage Allal El Fassi. Thèse $3^{\text {eme }}$ cycle, Univ. SMBA. Fès : 191p.

Jamet D. 1995. - Variations temporelles de l'activité de la phosphatase alcaline associée à différentes classes de taille du matériel particulaire au sein de trois lacs du massif central de degrés trophiques différents. Thèse de Doct. Univ. Blaise Pascal. ClermontFerrand : $180 \mathrm{p}$.

Jamet D., Aleya L. \& Devaux J. 1995. — Diel changes in the alkaline phosphatase activity of bacteria and phytoplankton in the hypereutrophe Villerest reservoir (Roanne, France). Hydrobiologia, 300/301 : 49-56.

Jamet D., Amblard C. \& Devaux J. 1997. - Seasonal changes in alkaline phosphatase activity of bacteria and microalgae in Lake Pavin (Massif Central, France). Hydrobiologia, 347 : 185-195.

Jansson M., Olsson H. \& Pettersson K. 1988. - Phosphatases : origin, characteristics and function in lakes. Hydrobiologia, 170 : 157-175.

Jones J.G. 1972. - Studies on freshwater microorganisms : phosphatase activity in Lake of differing degrees of eutrophication. J. Ecol., 60 : 777-791.

Kalinowska K. 1997. - Eutrophication processes in a shallow macrophyte dominated lake - alkaline phosphatase activity in Lake Luiknajno (Poland). Hydrobiologia, 342/343 : 395-399.

Lee S. \&.Furhman J.A. - 1987. Relationships between biovolume and biomass of naturally derived marine bacterioplankton. Appl. Environ. Microbiol., 53 : 1298-1303.

Legendre L. \& Watt W.D. 1971-1972. - On a rapid technique for plankton enumeration. Ann. Inst. Oceanogr., Paris, 58 : 173-177.

Lohman H. 1908. - Untersuchungen zur Feststellung des vollständigen Gehaltes des Meeres an Plankton. Wiss. Meeresunters, Abt. Kiel N. F., $10: 132$ - 170.

Loudiki M. 1990. - Etude limnologique d'un hydrosystème récemment aménagé dans la région de Marrakech (Maroc). Thèse de Doct. d'état, Univ. Aix Marseille III : 353p.

Olsson H. 1990. - Phosphatase activity in relation to phytoplankton composition and $\mathrm{pH}$ in Swedish Lake. Freshwat. Biol., 23 : 353-362.

Petterson K. 1980. - Alkaline phosphatase activity and algal surplus phosphorus as phosphorus deficiency indicator in Lake Erken. Arch. Hydrobiol., 89 : 54-87.

Porter K.G. \& Feig Y.S. 1980. - The use of DAPI for identifying and counting aquatic microflora. Limnol. Oceanogr., 25 : 943-948.

Reichardt W., Overbeck J. \& Steubing L. - 1967. Free dissolved enzymes in Lake waters. Nature, $216: 1345-1347$.

Rodier J. 1984. - Analyse de l'eau. Edit. Dunod, Paris : 1135 p.

SCOR-UNESCO. 1966. - Determination of photosynthetic pigments in sea water. UNESCO. Paris : $69 \mathrm{p}$.

Stewart A.J. \& Wetzel R.G. 1982. - Phytoplankton contribution to alkaline phosphatase activity. Arch. Hydrobiol., 93 : 265-271.

Utermöhl H. 1958. - Zur Vervollkommung der quantitative Phytoplankton-Methodik. Mitt. Int. Ver. Limnol., 9 : 1-38.

Wetzel R.G. 1981. - Long term dissolved and particulate alkaline phosphatase activity in a hardwater lake in relation to lake stability and phosphorus enrichment. Verh. Internat. Verein. Limnol., 21 : 369-381. 